\title{
Interação das espécies Echinochloa crusgalli e Pistia stratiotes para fitorremediação de Levonorgestrel
}

\author{
Phytoremediation of Levonorgestrel by interaction of species Echinochloa crusgalli and Pistia \\ stratiotes \\ R. T. P. Alves ${ }^{1}$; S. J. Andrade ${ }^{1 *} ;$ K. D. Fernandes ${ }^{2}$ \\ ${ }^{I}$ Instituto de Física e Química, Universidade Federal de Itajubá, 37500-903, Itajubá-MG, Brasil \\ ${ }^{2}$ Instituto de Recursos Naturais, Universidade Federal de Itajubá, 37500-903, Itajubá-MG, Brasil \\ *sandroandrade@unifei.edu.br
}

(Recebido em 12 de dezembro de 2019; aceito em 25 de abril de 2020)

\begin{abstract}
Os contaminantes emergentes provenientes dos avanços tecnológicos, industriais, agropecuários e farmacêuticos estão cada vez mais presentes na natureza, ameaçando à saúde humana e ao meio ambiente. O levonorgestrel (LNG) é um exemplo desses contaminantes que são encontrados em concentrações extremamente baixas, dificultando a remoção por técnicas convencionais de tratamentos de água e esgoto. Portanto, surgem métodos alternativos de remoção desses compostos, como o uso da fitorremediação. Essa técnica se baseia na remoção de contaminantes emergentes através de plantas. Diante disso, objetivou-se remover o LNG de solução aquosa através das espécies Echinochloa crusgalli e Pistia stratiotes juntas e separadamente. Utilizou-se 3 tratamentos de estudo ( $0,16 \mathrm{mg} \mathrm{L}^{-1}$ de Levonorgestrel), controle positivo (com hormônio, sem plantas) e controle negativo (com plantas, sem hormônio), seguido de 3 condições de plantas (apenas E. crusgalli; apenas $P$. stratiotes; ambas as espécies) em duas repetições, totalizando 30 experimentos. Analisou-se as soluções por Cromatografia Líquida de Alta Eficiência com detector de arranjo de diodos (HPLC/DAD). As determinações de LNG nas plantas foram realizadas através de Cromatografia acoplada ao Espectrômetro de Massas (GC/MS). No geral, os resultados obtidos apresentaram um desempenho satisfatório, pois na segunda semana todos os tratamentos de estudo obtiveram uma concentração de LNG abaixo do limite de quantificação do método $\left(0,025 \mathrm{mg} \mathrm{L}^{-1}\right)$. As análises macroscópicas das plantas não apresentaram diferenças significativas, indicando que ambas as espécies desenvolveram-se bem nesse meio. Assim, conclui-se que essas espécies podem ser utilizadas em programas de biomonitoramento e fitorremediação, tanto juntas quanto separadas para complemento de purificação de água e esgoto. Palavras-chave: Contaminantes Emergentes, Interferentes Endócrinos, HPLC.
\end{abstract}

Chemical compounds from technological, industrial, agricultural and pharmaceutical advances are increasingly present in the environment, threatening human health and the environment. Levonorgestrel (LNG) is an example of such contaminants that are found in extremely low concentrations, making it difficult to remove by conventional water and sewage treatment techniques. Therefore, alternative methods of removing these compounds have emerged. Phytoremediation is based on the removal of chemical compounds through plants. Therefore, it was aimed to remove the LNG from aqueous solution through the species Echinochloa crusgalli and Pistia stratiotes together and separately. Three study treatments $(0.16$ $\mathrm{mg} \mathrm{L}^{-1}$ ), positive control (with hormone, no species) and negative control (with plants, no hormone), followed by 3 plant conditions (E. crusgalli only; P. stratiotes; both species) in two repetitions. HPLC / DAD solutions were analyzed. Plant LNG determinations were performed by Mass Spectrometer-Coupled Chromatography (GC/MS). In general, the results obtained presented a satisfactory performance, because in the second week all study treatments obtained a LNG concentration below the method quantification limit $\left(0.025 \mathrm{mg} \mathrm{L}^{-1}\right)$. The macroscopic analyzes of the plants did not present significant differences, indicating that both species developed well in this environment. Thus, it can be concluded that these species can be used in biomonitoring and phytoremediation programs, both together and separately to complement water and sewage purification.

Keywords: Emerging Contaminants, Endocrine Disruptor, HPLC. 


\section{INTRODUÇÃO}

Recentemente, tem-se detectado muitos compostos químicos na água, no solo e no ar de todo o planeta destacando-se os compostos classificados como contaminantes emergentes (CEs), os quais ameaçam à saúde dos seres humanos e animais. De acordo com Collins e Silva (2011) [1], "CE é qualquer composto químico presente numa variedade de produtos comerciais, ou ainda qualquer microrganismo, que pode ser encontrado em matrizes ambientais e biológicas, que não são usualmente monitorados ou que ainda não possuem legislação regulatória correspondente, mas que apresentam risco potencial à saúde humana e ao meio ambiente". Esses CEs são encontrados em concentrações traço no meio ambiente, proporcionando dificuldades na purificação da água urbana [2].

Os CEs englobam mais de mil substâncias originadas de diversos produtos comerciais e industriais, porém, a maioria não possui legislação para controlar sua presença no meio ambiente, além de serem de difícil degradação naturalmente [3-6]. Essa preocupação se agrava com os efeitos negativos e/ou negligenciados que esses CEs causam nos ecossistemas e na saúde humana $[4,5]$.

Os CEs ocorrem de forma natural podendo ser encontrados em algumas espécies de plantas, mas provêm principalmente de ações antropogênicas como efluentes domésticos, industriais, hospitalares, atividades agropecuárias assim produtos farmacêuticos em geral [3-7].

Mesmo que esses CEs sejam detectados em baixas concentrações no ambiente, eles causam uma série de riscos à saúde humana e ao meio ambiente, principalmente os chamados Interferentes Endócrinos (IEs). Estima-se que os IEs compõem 1\% de todos os componentes químicos presentes no meio ambiente, e mesmo assim, alteram as funções do sistema endócrino ao imitar a ação de hormônios naturais, desencadeando doenças como câncer de mama e de próstata, anomalias nos órgãos genitais, deficiências cognitivas e neurológicas, infertilidade, aborto espontâneo, puberdade precoce e obesidade, além de causar feminização de peixes e problemas na reprodução de alguns seres vertebrados [8-14].

Os contraceptivos são IEs amplamente usados. Segundo a Pesquisa Nacional de Saúde (PNS) em 2013, as mulheres compunham 51,9\% da população brasileira, sendo as principais usuárias dos serviços de saúde. No mesmo ano $61,1 \%$ das mulheres com idade entre 18 e 49 anos que eram sexualmente ativas e que menstruavam fizeram uso de métodos contraceptivos [15]. Esses fármacos contraceptivos para terapias de reposição hormonal, para tratamento de neoplasias e outros produtos farmacêuticos possuem os chamados estrogênios sintéticos, os quais são esteroides que tiveram suas estruturas moleculares modificadas para possuírem alto potencial estrogênico, causando alterações no corpo humano por agirem diretamente no sistema endócrino [3].

Os estrogênios são uma classe de compostos que não é eliminada totalmente pelas estações de tratamento de água e esgoto, e como esses compostos são fisiologicamente ativos em baixas concentrações, é relevante o estudo do comportamento de degradação desses contaminantes nessas estações e no ambiente [3].

Dentre essa classe de hormônios sintéticos, encontra-se o Levonorgestrel (LNG) ou 13ß-etil17 $\alpha$-etinil-17 $\beta$-hidroxigon-4-en-3-ona (CAS 797-63-7). É uma progesterona sintética derivada da testosterona e o enantiômero levógiro ativo do norgestrel. Os principais metabólitos excretados pelo organismo humano depois da ingestão do LNG são $3 \alpha, 5 \beta$-tetraidrolevonorgestrel, $3 \alpha, 5 \alpha-$ tetraidrolevonorgestrel e 16- $\beta$-hidroxinorgestrel [9-12]. Esse hormônio sintético tem sido detectado em baixas concentrações $\left(\mu \mathrm{L}^{-1} \mathrm{e} \mathrm{ng} \mathrm{L}^{-1}\right)$ nas estações de tratamento de esgoto, águas superficiais e subterrâneas e sedimentos, mostrando-se persistente e estável no meio ambiente, não sendo removido com eficiência por tratamentos químicos e biológicos convencionais e afetando diretamente a vida de seres aquáticos e do ser humano [10].

Para remediar as áreas contaminandas, aplicam-se de técnicas para remover, conter ou reduzie das concentrações de contaminantes, como explica na Resolução do Conselho Nacional de Meio Ambiente (CONAMA) número 420 (2009) [16]. Métodos alternativos como os processos oxidativos avançados (POAs), processos eletroquímicos e nanofiltração vêm sendo amplamente estudados para remover esses compostos. Entretanto, as principais desvantagens desses métodos 
é a geração de produtos de degradação mais tóxicos que os compostos originais e o alto custo de implementação dessas técnicas [4,7].

Um método que vem ganhando destaque na remoção de CEs faz o uso de certos materiais conhecidos como biossorventes. Essa técnica possui alta eficiência e seletividade, baixo custo e simplicidade, além de permitir reciclagem e reutilização dos biossorventes [7]. Esse grupo engloba toda a biomassa ativa (com atividade metabólica) ou inativa (sem atividade metabólica) de qualquer matéria de origem vegetal ou animal, tais como resíduos agroindustriais, bactérias, algas, fungos, leveduras e vegetais [17].

Nesse sentido, a fitorremediação utiliza vegetais e seus microorganismos como biossorventes para remover, degradar, estabilizar ou desintoxicar o meio ambiente de contaminantes orgânicos e inorgânicos, recuperando áreas poluídas $[10,14,18]$. Suas vantagens incluem aplicabilidade de processos biológicos in-situ e/ou ex-situ, baixo impacto ambiental, fácil implementação, remediação simultânea de vários contaminantes, extensa aplicação, contenção de riscos e aumento da qualidade do ambiente $[18,19]$. Portanto, mesmo que a biomassa contaminada ainda seja um problema para tratamento e descarte, a fitorremediação é ecologicamente viável [20].

As plantas aquáticas têm demonstrado resultados excelentes na fitorremediação [21] podendo destacar a Echinochloa crusgalli (E. crusgalli) e a Pistia stratiotes (P. stratiotes). A E. crusgalli, popularmente conhecida como capim-arroz, é uma gramínea daninha comum nos campos de arroz que cresce anualmente, naturalmente e em abundância, mesmo em áreas contaminadas por metais pesados, como o arsênio, porém, pouco se sabe de seu potencial fitorremediador [22]. Muitos estudos têm sido feitos com a $P$. stratiotes, mais conhecida como alface d'água, uma macrófita aquática encontrada em todo o planeta, principalmente em áreas tropicais e sub-tropicais. Os resultados têm sido satisfatórios na remoção de metais pesados de ambientes aquáticos [23, 24].

Sabendo do potencial dessas espécies para a fitorremediação objetivou-se analisar a sorção do hormônio LNG em solução aquosa utilizando as espécies $E$. crusgalli e P. stratiotes juntas e separadamente.

\section{MATERIAL E MÉTODOS}

\subsection{Condições cromatográficas}

As soluções foram preparadas e analisadas por Cromatografia Líquida de Alta Eficiência (HPLC) Modelo Infinity 1260 - Agilent Technologies®, equipado com um detector de arranjo de diodos (DAD), uma bomba quaternária e um injetor automático, otimizando a metodologia desenvolvida por Qiao et al. (2009) [8]. A eluição do LNG se deu em uma coluna cromatográfica Eclipse Plus C8 (fase reversa) por uma fase móvel (FM) de mistura 54\% Acetonitrila (ACN) e $46 \%$ água (v/v) no modo isocrático. A vazão foi de $0,500 \mathrm{~mL} \cdot \mathrm{min}^{-1}$ durante $15 \mathrm{~min}$. O analito foi detectado na região do ultravioleta em um comprimento de onda de $242 \mathrm{~nm}$, sendo determinado pela área do pico identificado pelo tempo de retenção do composto. As condições cromatográficas estão sintetizadas na Tabela 1.

Tabela 1: Condições cromatográficas empregadas na análise por HPLC/DAD.

\begin{tabular}{cc}
\hline Parâmetros & Condições \\
\hline Coluna cromatográfica & ZORBAX® Eclipse Plus C8 $(4,6 \times 150 \mathrm{~nm} ; 5 \mu \mathrm{m})$ \\
Fase móvel (modo isocrático) & $\mathrm{ACN}: \mathrm{H}_{2} \mathrm{O}(54: 46 \mathrm{v} / \mathrm{v})$ \\
Vazão & $0,5 \mathrm{~mL} \mathrm{~min}{ }^{-1}$ \\
Volume de injeção & $10 \mu \mathrm{L}$ \\
Temperatura da coluuna & $45 \pm 1^{\circ} \mathrm{C}$ \\
Tempo de corrida & $15 \mathrm{~min}$ \\
Detector DAD & $\lambda=242 \mathrm{~nm}$ \\
Tempo de retenção & $8,85 \mathrm{~min}$ \\
\hline
\end{tabular}

Fonte: Adaptada de QIAO et al. (2009) [8] 


\subsection{Curva analítica}

Preparou-se uma solução estoque de $10,0 \mathrm{mg} \cdot \mathrm{L}^{-1}$ de um padrão de LNG (European Pharmacopoeia Reference Standart® - Lote: L0551000) em ACN. Essa solução foi diluída em oito balões volumétricos de $10 \mathrm{~mL}$ (faixa de linearidade: $0,025-1,0 \mathrm{mg} \mathrm{L}^{-1}$ ) e o menisco foi aferido com solução nutritiva de Hoagland e Arnon (1950) [25] nº 2 modificada, com $40 \%$ da força iônica, em água deionizada. As soluções foram filtradas e transferidas para vials para análise por HPLC/DAD. A curva analítica foi construída pelo software Microsoft Excel@.

\subsection{Preparo da solução de LNG}

Preparou-se três soluções de LNG a partir de um anticoncepcional comercial (Ciclo $21^{\circledR}$ ) na concentração de $0,2 \mathrm{mg} \mathrm{L}^{-1}$, sendo três tratamentos de estudo, um controle positivo (com o hormônio e sem as espécies) e um controle negativo (com as plantas e sem o hormônio). Para isso, macerou-se 6 comprimidos (cada um com $0,15 \mathrm{mg}$ de Levonorgestrel) para cada solução, transferiu-os separadamente para um béquer de $2000 \mathrm{~mL}$ e adicionou-se água deionizada para dissolver e solubilizar o pó. Realizou-se filtração à vácuo, para separar o LNG dos excipientes do comprimido. Posteriormente, cada solução foi deixada em banho ultrassônico durante 6 min. Após, transferiu-se a solução obtida para um balão volumétrico de $5 \mathrm{~L}$ com solução nutritiva de Hoagland e Arnon (1950) n`2 [25] modificada, com 40\% da força iônica, e acertou-se o menisco com água destilada.

\subsection{Instalação do experimento}

As sementes de E. crusgalli foram obtidas no Instituto Rio-grandense de Arroz - RS e levadas para a casa-de-vegetação do Instituto de Recursos Naturais da Universidade Federal de Itajubá para a germinação. Já as mudas de $P$. stratiotes foram obtidas de lagos da região de Itajubá (MG) e levadas para a casa-de-vegetação para propagação até a $3^{a}$ geração clonal, a fim de remover qualquer resíduo de LNG que possa estar contida nas plantas. Essa metodologia é usual quando se trabalha com fitorremediação como no trabalho de Pereira et al. (2017) [26] em que publicou o trabalho "Anatomical and physiological modifications in water hyacinth under cadmium contamination" na revista Journal of Applied Botany and Food Quality com uma metodologia semelhante. O princípio é retirado da metodologia da cultura de tecidos de plantas em que a ausência de tecidos vasculares no meristema dificulta o transporte de partículas de outras partes da planta para esta estrutura, gerando assim plantas livres de contaminações.

Mudas de E. crusgalli com 30 dias de desenvolvimento e de P. stratiotes foram selecionadas quanto à uniformidade do comprimento e massa, sendo de aproximadamente $3 \mathrm{~g}$ de E. crusgalli e $12 \mathrm{~g}$ de $P$. stratiotes. As plantas foram transferidas para bandejas de alumínio de $1000 \mathrm{~mL}$ contendo $500 \mathrm{~mL}$ das soluções do LNG cobertos com papel alumínio. O nível de solução nas bandejas foi complementado com solução nutritiva sem LNG sempre que necessário. $O$ experimento foi montado com duas repetições em delineamento inteiramente casualizado (DIC) com uma concentração de $0,16 \mathrm{mg} \mathrm{L}^{-1}$ de LNG seguida de 2 controles (negativo - com planta e sem LNG; positivo - sem planta e com LNG na concentração de $0,14 \mathrm{mg} \mathrm{L}^{-1}$ ) e 3 condições de plantas (apenas mudas de E. crusgalli; apenas mudas de P. stratiotes; ambas as espécies) realizados em duplicata e totalizando 30 experimentos. A Figura 1 ilustra o processo de preparo das soluções e montagem das bandejas. 


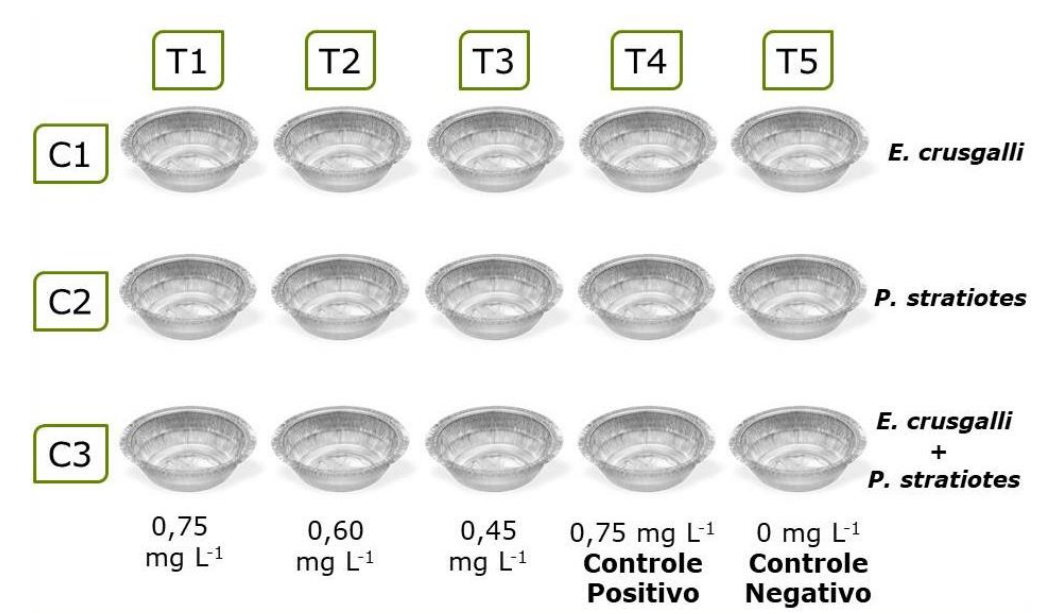

Figura 1: Representação dos tratamentos preparados para estudo de fitorremediação utilizando-se Echinochloa crusgalli e Pistia stratiotes.

\subsection{Análises químicas}

As determinações de LNG nas soluções foram realizadas retirando-se alíquotas a cada sete dias com uma seringa de vidro e filtrada através de uma membrana de poliamida $(0,45 \mu \mathrm{m})$ para vials e analisadas em HPLC/DAD. Retirou-se uma alíquota inicial de cada solução para determinar a concentração real de LNG em solução. Após 7 dias, retirou-se uma alíquota de cada ensaio, e novamente após 14 dias. Finalizado o experimento, as plantas foram colhidas, lavadas e congeladas em freezer a $-3^{\circ} \mathrm{C}$.

As determinações de LNG nas plantas foram realizadas através de Cromatografia Gasosa (Agilent Technologies ${ }^{\circledR}$ - Sistema 7890A) acoplada ao Espectrômetro de Massas (GC/MS) (Agilent Technologies® - Modelo 5975C XL MSD inerte com detector de eixo triplo) com amostrador automático (Agilent Technologies® - Modelo 7693). Os procedimentos foram baseados na metodologia desenvolvida por Li et al. (2014) [10]. Analisou-se as soluções também em HPLC/DAD.

Preparou-se uma amostra da parte aérea (com combinação das espécies) e uma amostra da raiz (apenas E. crusgalli). Para isso, pesou-se e macerou-se com sílica gel 60 da Sorbline Tecnologie ${ }^{\circledR}$ separadamente as plantas ainda congeladas com o auxílio de um pistão, almofariz de porcelana. Após, adicionou-se $20 \mathrm{~mL}$ de ACN e transferiu-se para um tubo Falcon. Os excipientes foram descartados e os sobrenadantes foram filtrados e transferidos para um balão de vidro de fundo redondo de $50 \mathrm{~mL}$. Os balões foram acoplados em um rotoevaporador (IKA® 10 digital) a uma temperatura de $50^{\circ} \mathrm{C}$ com $100 \mathrm{rpm}$ durante $30 \mathrm{~min}$ (parte aérea) e 15 min (raiz). Após entrar em equilíbrio com a temperatura ambiente, adicionou-se $2 \mathrm{~mL}$ de metanol da Cromoline ${ }^{\circledR}$ em cada balão para dissolver o precipitado e filtrou-se com filtro de membrana de poliamida (poros de $0,45 \mu \mathrm{m})$.

As amostras foram transferidas para vials para análise por HPLC/DAD e por GC/MS (Agilent Technologies ${ }^{\circledR}$ - Modelo 7890A) com amostrador automático (Agilent Technologies ${ }^{\circledR}$ - Modelo 7693) acoplado à um espectrômetro de massas (Agilent Technologies® - Modelo 5975C inert XL MSD) com detector quadrupolo. A eluição do LNG por GC/MS se deu em uma coluna cromatográfica capilar de baixo sangramento de $30 \mathrm{~m}$ de comprimento, $0,250 \mathrm{~mm}$ de diâmetro interno com $0,25 \mu \mathrm{m}$ de fase estacionária (filme interno) de composição $5 \%$ fenil - $95 \%$ metilpolissiloxano (HP-5MS) apolar. A vazão do gás de arraste (FM - Hélio) por GC/MS foi de $1,000 \mathrm{~mL} \mathrm{m^{-1 }}$ com uma temperatura no injetor de $250^{\circ} \mathrm{C}$ e volume de injeção de $1 \mu \mathrm{L}$ no modo splitless (injeção sem divisão de fluxo). As temperaturas da fonte de íons, do quadrupolo e da interface foram de $230^{\circ} \mathrm{C}, 150^{\circ} \mathrm{C}$ e $280^{\circ} \mathrm{C}$, respectivamente. A injeção no espectro de massas se deu no modo de aquisição SCAN (fornece a máxima informação qualitativa ao coletar todos os íons, produzindo espectros completos das moléculas) e solvente delay de 3 min. 


\section{RESULTADOS E DISCUSSÃO}

\subsection{Curva analítica}

A curva analítica utilizada na determinação do LNG está representada na Figura 2 e explica $99,48 \%$ da variabilidade dos dados.

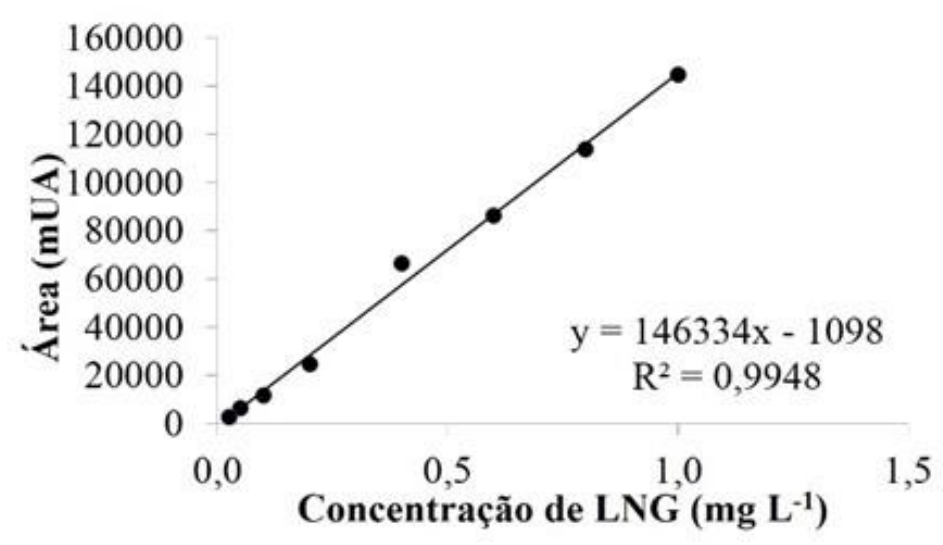

Figura 2: Curva analítica para determinação de LNG em solução aquosa.

O limite de quantificação (LQ) do método corresponde ao padrão de calibração da menor concentração que se difere do branco, ou seja, o LQ fornece o menor valor de concentração do analito que pode ser quantificado pelo método utilizado. No método de determinação sinal/ruído, o LQ é obtido numa proporção do sinal de 10 vezes o tamanho do ruído (10:1) [27]. Assim, o valor de LQ encontrado foi de $0,025 \mathrm{mg} \mathrm{L}^{-1}$.

\subsection{Seletividade e tempo de retenção}

O método mostrou-se seletivo, uma vez que o pico do analito é visível, sendo em torno de 8,85 min, sem interferência de outros componentes da solução, como é possível observar na Figura 3.
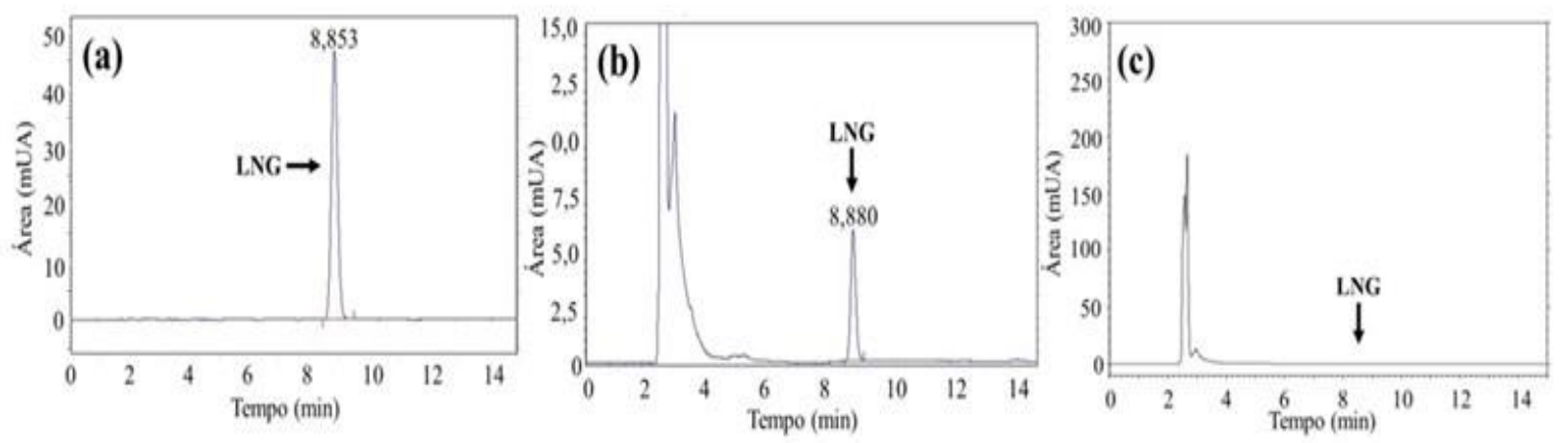

Figura 3: Cromatogramas por HPLC/DAD do (a) padrão de $L N G\left(10,0 \mathrm{mg} \mathrm{L}^{-1}\right)$ em ACN, do (b) padrão de LNG (1,0 $\left.\mathrm{mg} \mathrm{L}^{-1}\right)$ diluído em solução nutritiva e do (c) Branco (solução nutritiva).

\subsection{Análise da fitorremediação}

Os valores da concentração dos tratamentos testados se encontram na Figura 4. O menor valor no controle positivo no tempo zero pode estar relacionado com a dificuldade de dissolver o LNG em água, visto que a solubilidade dele é de $2,1 \mathrm{mg} \mathrm{L}^{-1}\left(25^{\circ} \mathrm{C}\right)$. 


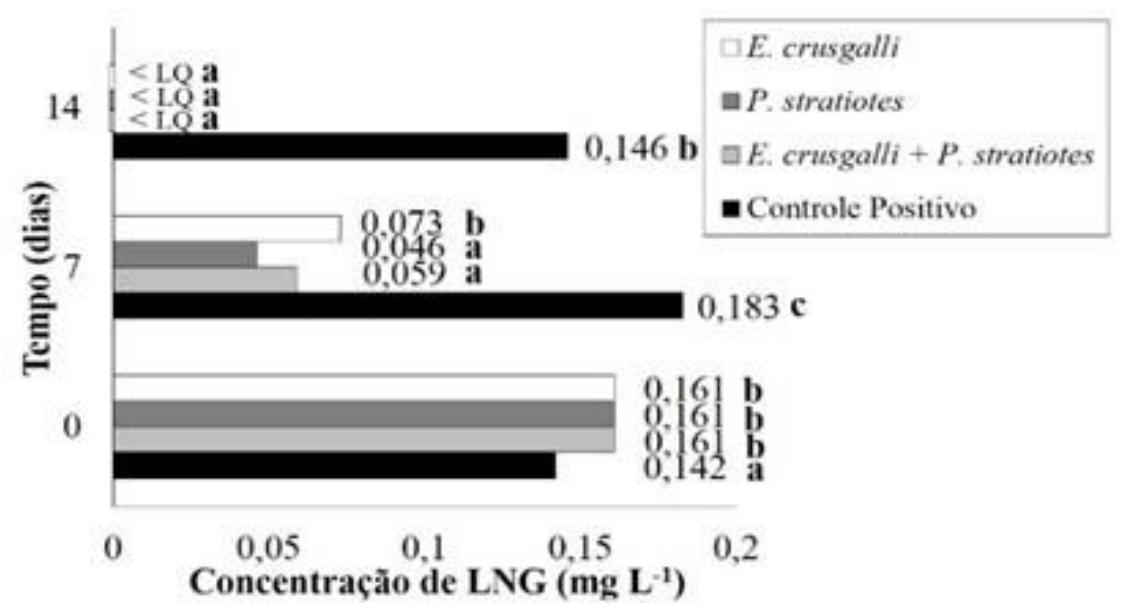

Figura 4: Média das concentrações de $L N G\left(m g L^{-1}\right)$ pelo tempo de remediação em relação às espécies utilizadas nos tratamentos *Médias seguidas pela mesma letra não diferem entre si estatisticamente pelo teste de Skott-knot a $5 \%$ de confiança.

Após 7 dias observou-se uma remoção de $71,4 \%$ para o tratamento utilizando $P$. stratiotes e $63,4 \%$ para o tratamento que continha ambas as espécies, sendo estatisticamente iguais. Já a $E$. crugalli teve um desempenho inferior após 7 dias removendo 54,6\% do LNG. Porém após 14 dias de experimentação não houve diferença estatística entre as plantas utilizadas $(\mathrm{p}=0,52)$. Tanto a $P$. stratiotes como a $E$. crusgalli, juntas ou isoladas, no presente trabalho podem ser utilizadas para remediação do hormônio presente em ambientes aquáticos. Isso pode ter ocorrido devido ao sistema radicular fasciculado das duas espécies, que ocupa áreas semelhantes para a absorção. Até 14 dias observou-se uma redução acima de $84 \%$ na concentração do hormônio com as plantas utilizadas em todos os tratamentos. Isso indica que ambas as espécies possuem excelente efeito fitorremediador, removendo o LNG do meio contaminado em até 14 dias.

O desenvolvimento das espécies no meio contaminado pelo LNG não foi afetado, pois não houve diferenças visuais significativas entre o comprimento e a coloração das espécies nos tratamentos, o que comprova o potencial fitorremediador de ambas as espécies na remoção e biomonitoramento de LNG nas estações de tratamento de água e esgoto.

De acordo com os cromatogramas das soluções com $0,16 \mathrm{mg} \mathrm{L}^{-1}$ de LNG nas três condições, aos 14 dias, o LNG foi removido efetivamente, como pode ser observado pela Figura 5. Da primeira para a segunda análise os valores da concentração dos tratamentos caíram bruscamente e da segunda para a terceira análise os valores foram menores que o LQ.
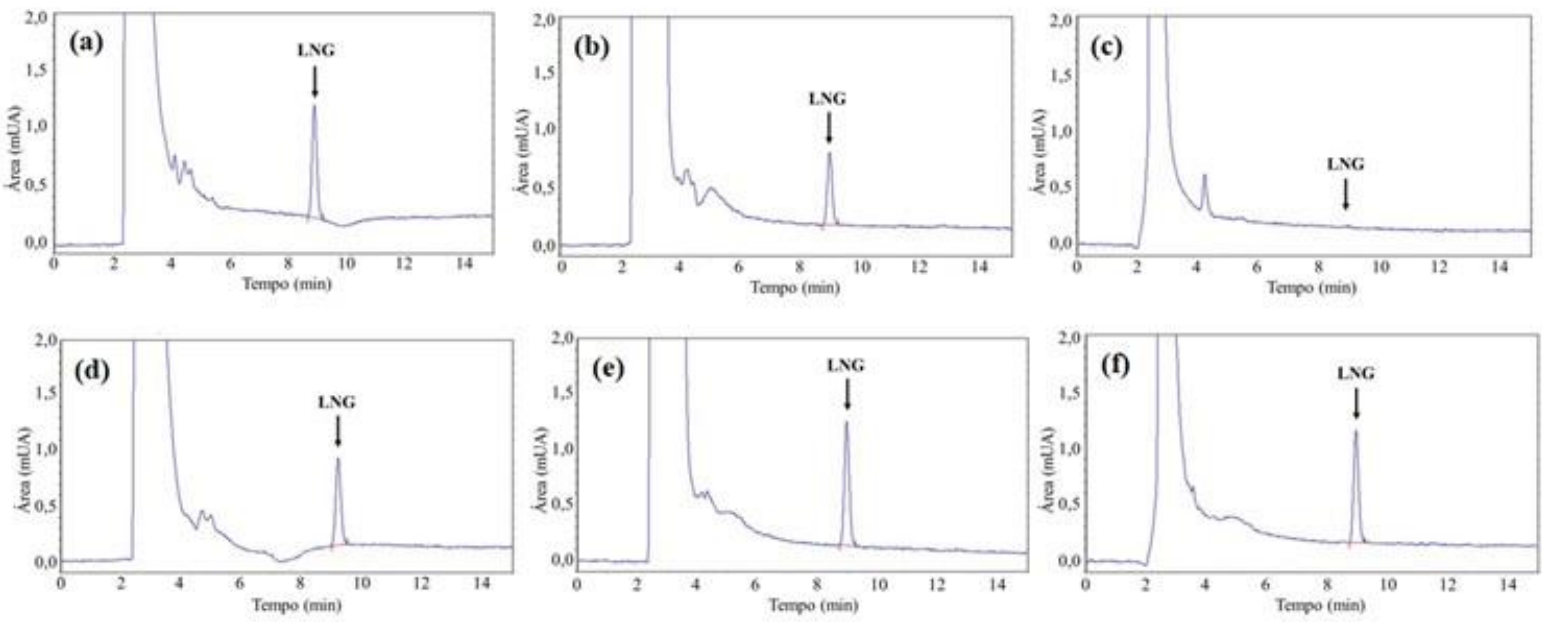

Figura 5: Cromatogramas por HPLC/DAD das soluções com 0,16 $\mathrm{mg} \mathrm{L}^{-1}$ de LNG (a) dia 0 para todos os tratamentos de estudo, (b) dia 7 do tratamento apenas com P. stratiotes e (c) dia 14 do tratamento com ambas as espécies, e do controle positivo (sem planta e com LNG) na concentração de $0,14 \mathrm{mg} \mathrm{L}^{-1}(\mathrm{~d})$ dia 0 , (e) dia 7 e (f) dia 14. 
De fato, Li et al. (2014) [10] determinaram uma meia-vida de 13 dias para o LNG utilizando as espécies Cyperus alternifolius e Eichhornia crassipes e observaram também que houve uma leve remoção do contaminante no controle positivo, possivelmente pela radiação ultravioleta provinda do sol e/ou devido aos microorganismos que se desenvolveram no meio, como fungos e algas [28, 29]. Como o esperado, o controle negativo não apresentou nenhum pico nos cromatogramas obtidos e por isso as imagens não foram utilizadas.

\subsection{Análise do LNG nas espécies contaminadas}

A Figura 6 ilustra o pico do Levonorgestrel ao ser analisado por GC/MS tanto do padrão quanto das soluções após o processo de extração.
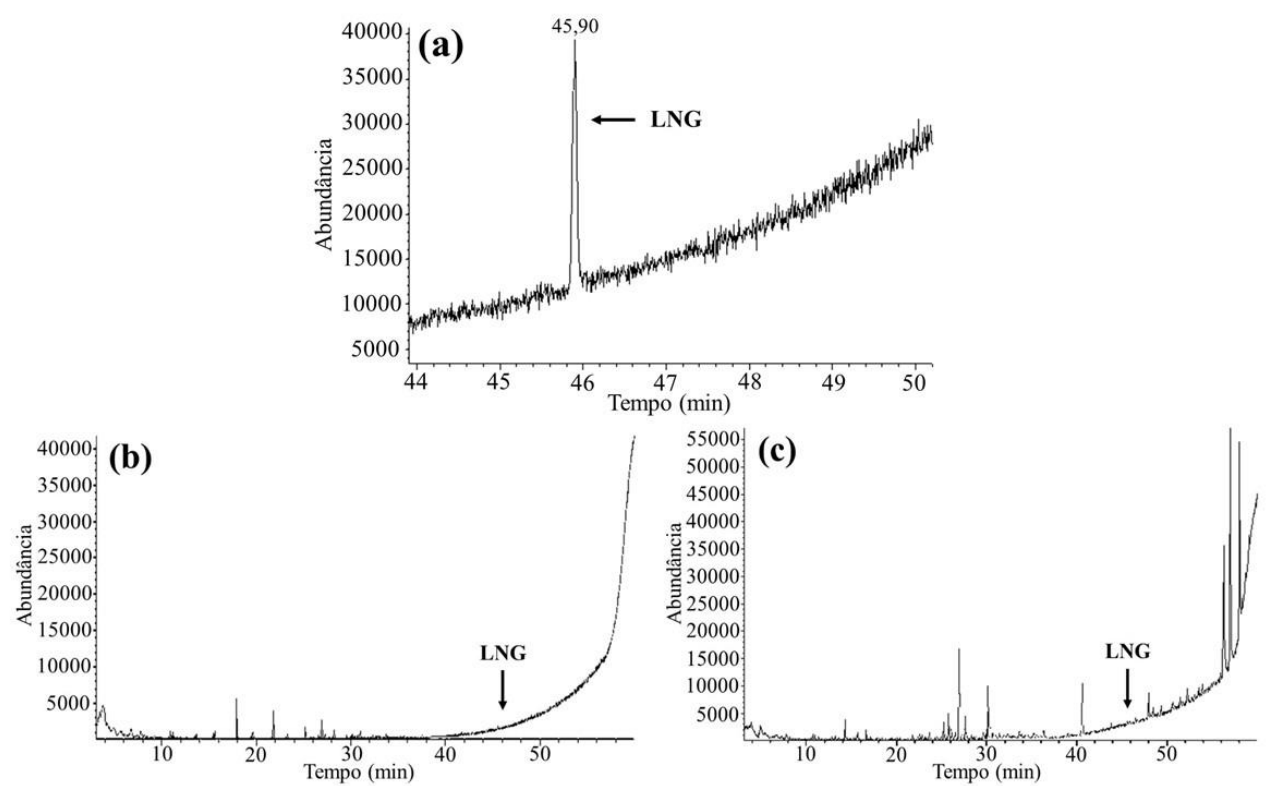

Figura 6: Cromatogramas (a) do padrão de $10 \mathrm{mg} \mathrm{L} \mathrm{L}^{-1}$ em ACN de LNG, (b) da solução após extração de LNG da parte aérea do tratamento com ambas as espécies e (c) da solução após extração da raiz do tratamento apenas com a espécie E. crusgalli por GC/MS.

O pico referente ao contaminante, próximo de 45,90 min, não foi observado nas amostras, indicando que o hormônio não pôde ser detectado na solução analisada da extração do LNG das espécies contaminadas. No entanto, outros picos referentes a componentes da planta mostraramse bastante visíveis nos cromatogramas, como era o esperado. Tais compostos encontrados na parte aérea das plantas, como o ginsenol, e nas raízes, como o campesterol, o stigmasterol e o sitosterol, estão representados na Figura 7.
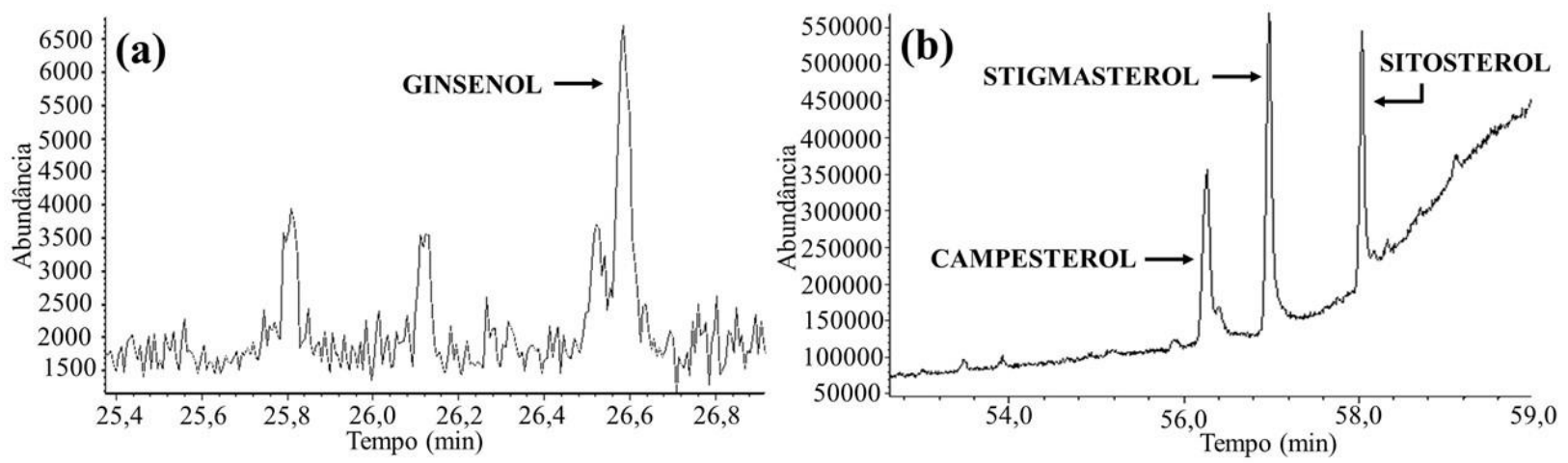

Figura 7: Cromatogramas (a) da solução após extração de LNG da Parte Aérea do tratamento contendo ambas as espécies e (b) da solução após extração da Raiz do tratamento contendo apenas a espécie E. crusgalli por GC/MS. 
De acordo com os dados obtidos pelos cromatogramas por GC/MS e HPLC/DAD (Figura 8) não há como afirmar como que o LNG interagiu com as espécies estudadas, pois não houve pico referente ao LNG nos cromatogramas para a parte aérea e muito baixo para a raiz. Todavia, de acordo com Li et al. (2014) [10], a acumulação por adsorção do LNG nas raízes das espécies Cyperus alternifolius e Eichhornia crassipes foi baixo, assim como as concentrações nas folhas da espécie Cyperus alternifolius, indicando que a fitovolatilização não era um caminho de eliminação provável, mesmo que haja absorção do contaminante pela planta. Portanto, o principal mecanismo que ocorreu foi o de fitodegradação, onde o contaminante é metabolizado através da raiz e seus microorganismos. Outra indicação desse mecanismo é que o LNG é lipofílico, ou seja, como os tecidos das raízes consistem principalmente de conteúdo lipídico, o LNG tem uma tendência a permanecer e ser convertido na raiz, pois é na raiz que ocorre o primeiro contato do analito com a planta [10].
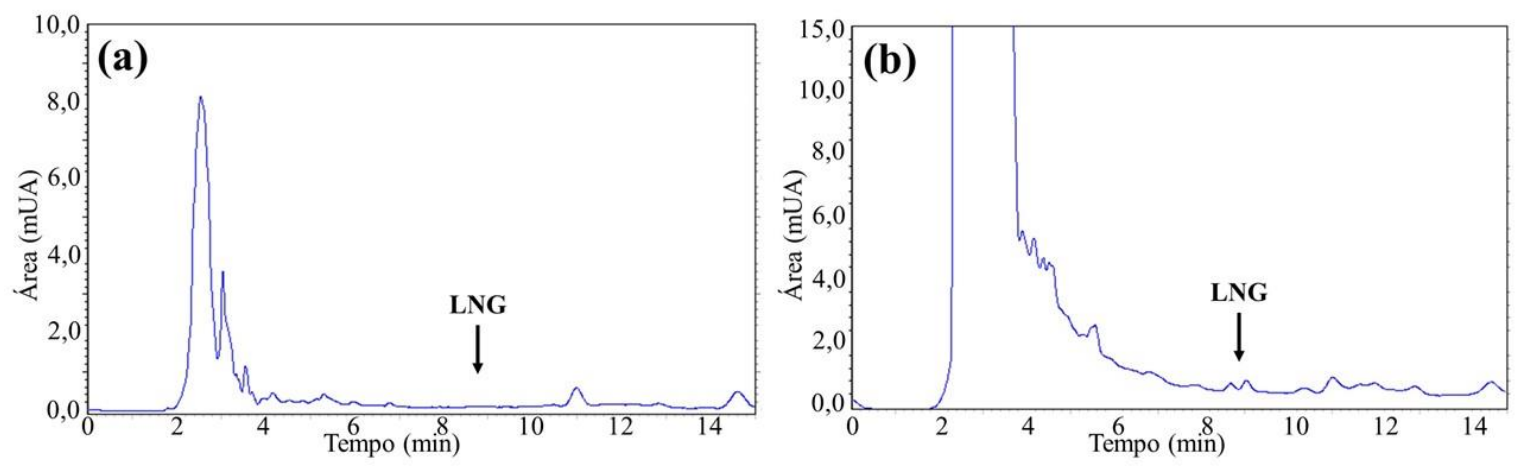

Figura 8: Cromatogramas (a) da solução após extração de LNG da Parte Aérea do tratamento contendo ambas as espécies e (b) da solução após extração da Raiz do tratamento contendo apenas a espécie E. crusgalli por HPLC/DAD.

O comportamento de acumulação do analito na superfície das raízes de macrófitas e sua migração para os tecidos das raízes, com dificuldade de translocação para a parte aérea, também foi observado na fitorremediação de fármacos por outras macrófitas [10, 30, 31]. Sendo assim, é bem provável que o LNG adsorvido pelas espécies em estudo esteja na forma de metabólitos na raiz, onde foi transformado e degradado gradualmente pelas plantas. Contudo, necessita-se de um desenvolvimento mais aprofundado da metodologia para detecção do mesmo nas espécies $E$. crusgalli e $P$. stratiotes.

\section{CONCLUSÃO}

As espécies E. crusgalli e $P$. stratiotes são potenciais agentes fitorremediadores em aplicações em programas de fitorremediação no tratamento complementar em estações de água e esgoto para a sorção do hormônio LNG. Essas espécies podem ser utilizadas tanto juntas como separadas, não havendo diferença estatística nas duas condições até 14 dias de experimentação. O período de 14 dias foi o suficiente para uma remoção eficiente do LNG em ambientes aquáticos nas concentrações testadas. As espécies testadas foram as principais contribuintes para a remoção do contaminante. Como trabalhos futuros, a montagem de um banco de macrófitas com essas e outras espécies é uma proposta para acelerar o tempo de fitorremediação do LNG em tratamentos de água e esgoto. 


\section{AGRADECIMENTOS}

À UNIFEI, por disponibilizar a estrutura para o estudo. Ao Instituto Rio-grandense de Arroz/RS, por fornecer as sementes de E. crusgalli. À Rede Mineira de Química (RQ-MG) e à FAPEMIG (Project: CEX-RED-00010-14).

\section{REFERÊNCIAS BIBLIOGRÁFICAS}

1. Collins $\mathrm{CH}$, Silva CGA. Aplicações de cromatografia líquida da alta eficiência para estudo de poluentes orgânicos emergentes. Quim Nova. 2011 Feb;34(4):665-676, doi: 10.1590/S0100-40422011000400020

2. Penã-Gusmán C, Ulloa-Sánchez S, Mora K, Helena-Bustos R, Lopez-Barrera E, Alvarez J, RodriguezPinzón. Emerging pollutants in the urban water cycle in Latin America: A review of the current literature. J Environ Manage. 2019 Feb;237:408-423, doi: 10.1016/j.jenvman.2019.02.100

3. Montagner CC, Vidal C, Acayaba RD. Contaminantes emergentes em matrizes aquáticas do Brasil: cenário atual e aspectos analíticos, ecotoxicológicos e regulatórios. Quim Nova. 2017 Jun;40(9):10941110, doi: 10.21577/0100-4042.20170091

4. Orona-Návar C, García-Morales R, Rubio-Govea R, Mahlknecht J, Hernandez-Aranda RI, Ramírez JG, Nigam KDP, Ornelas-Soto N. Adsorptive removal of emerging pollutants from groundwater by using modified titanate nanotubes. J Environ Chem Eng. 2018 Aug;6(4):5332-5340, doi: 10.1016/j.jece.2018.08.010

5. Mijangos L, Ziarrusta H, Ros O, Kortazar L, Fernandez LA, Olivares M, Zuloaga O, Prietro A, Etxebarria N. Occurrence of emerging pollutants in estuaries of the Basque Country: Analysis of sources and distribution, and assessment of the environmental risk. Water Res. 2018 Dec;147:152-163, doi: 10.1016/j.watres.2018.09.033

6. Teodosiu C, Gilca AF, Barjoveano G, Fiore S. Emerging pollutants removal through advanced drinking water treatment: A review on processes and environmental performances assessment. J Clean Prod. 2018 Oct;197(1):1210-1221, doi: 10.1016/j.jclepro.2018.06.247

7. Martín J, Orta MM, Medina-Carrasco S, Santos JL, Aparicio I, Alonso E. Removal of priority and emerging pollutants from aqueous media by adsorption onto synthetic organo-funtionalized high-charge swelling micas. Environ Res. 2018 Jul;164:488-494, doi: 10.1016/j.envres.2018.03.037

8. Qiao Y, Yang H, Wang B, Song J, Den A. Preparation and characterization of an immunoaffinity chromatography column for the selective extraction of trace contraceptive drug levonorgestrel from water samples. Talanta. 2009 Nov;80(1):98-103, doi: 10.1016/j.talanta.2009.06.029

9. Walker SD, McEldowney S. Molecular docking: a potential tool to aid ecotoxicity testing in environmental risk assessment of pharmaceuticals. Chemosphere. 2013 Nov;93(10):2568-2577, doi: 10.1016/j.chemosphere.2013.09.074

10. Li G, Zhai J, He Q, Zhi Y, Xiao H, Rong J. Phytoremediation of levonorgestrel in aquatic environment by hydrophytes. Int J Environ Sci. 2014 Sep;26(9):1869-1873, doi: 10.1016/j.jes.2014.06.030

11. Runnals TJ, Beresford N, Kugathas S, Casaluci LM, Scholze M, Scott AP, Sumpter J P. From single chemicals to mixtures - Reproductive effects of levonorgestrel and ethinylestradiol on the fathead minnow. Aquat Toxicol. 2015 Dec;(169):152-167, doi: 10.1016/j.aquatox.2015.10.009

12. Cirrincione LR, Penchala SD, Scarsi KK, Podany AT, Winchester LC, Back DJ, Khoo SH, Fletcher CV, Siccardi M, Else LJ. Development, validation and utilization of a highly sensitive LC-MS/MS method for quantification of levonorgestrel released from a subdermal implant in human plasma. $\mathbf{J}$ Chromatogr B. 2018 May;(1084):106-112, doi: 10.1016/j.jchromb.2018.03.023

13. DeWitt JC, Patisaul, HB. Endocrine disruptors and the developing immune system. Curr Opin Toxicol. 2018 Aug;10:31-36, doi: 10.1016/j.cotox.2017.12.005

14.Zhang S, Mo J, Wang Y, Ni C, Li, X, Zhu Q, Ge RS. Endocrine disruptors of inhibiting testicular 3 $\beta$ hydroxysteroid dehydrogenase. Chem Biol Interact. 2019 Apr;303:90-97, doi: 10.1016/j.cbi.2019.02.027

15. IBGE - Instituto Brasileiro de Geografia e Estatística. Pesquisa Nacional de Saúde, 2013 - Ciclos de Vida. Rio de Janeiro: IBGE, 2015. ISBN 978-85-240-4351-2

16. Resolução No 420, 28 de Dezembro de 2009. DOU n 249, 30/12/2009, p.81-84.

17. Labuto G, Silva LR, Silva HDT, Carrilho ENVM, Gonçalves NS, Rodrigues CA. Efficient degradation of solid yeast biomass from ethanol industry by Fenton and UV-Fenton processes applying multivariate analysis. Rev Ambient Água. 2017 Nov/Dec;12(6):946-963, doi: 10.4136/ambi-agua.2124

18. Odoh CK, Zabbey N, Sam K, Eze CN. Review: Status, progress and challenges of phytoremediation an Africa scenario. J Environ Manage. 2019 May;237:365-378, doi: 10.1016/j.jenvman.2019.02.090 
19. Mejía PVL, Andreoli FN, Andreoli CV, Serrat BM. Metodologia para seleção de técnica de fitorremediação em áreas contaminadas. Rev Bras Ci Ambient. 2014 Mar;(31):97-104.

20. Sharma S, Singh B, Manchanda VK. Phytoremediation: role of terrestrial plants and aquatic macrophytes in the remediation of radionuclides and heavy metal contaminated soil and water. Environ Sci Pollut Res Int. 2015 Jan;22(2):946-962 doi: 10.1007/s11356-014-3635-8

21. Jesus TB, Souza SS, Santos LTSO, Aguiar WM. Avaliação da potencialidade de utilização de espécies de macrófitas como acumuladoras de metais pesados. Rev Virtual Quim. 2015 May;7(4):1102-1118, doi: 10.5935/1984-6835.20150061

22. Sultana R, Kobayashi K. Potential of barnyard grass to remediate arsenic-contaminated soil. Weed Biol Manag. 2011 Mar;11(1):12-17, doi: https://doi.org/10.1111/j.1445-6664.2011.00400.x

23. Farnese FS, Oliveira JA, Lima FS, Leão GA, Gusman GS, Silva LC. Evaluation of the potential of Pistia stratiotes L. (water lettuce) for bioindication and phytoremediation of aquatic environments contaminated with arsenic. Braz J Biol. 2014 Aug;74(3):103-112, doi: 10.1590/1519-6984.01113

24. Hanks NA, Caruso JA, Zhang P. Assessing Pistia stratiotes for phytoremediation of silver nanoparticles and $\operatorname{Ag}(\mathrm{I})$ contaminated waters. J Environ Manage. 2015 Dec;164:41-45, doi: 10.1016/j.jenvman.2015.08.026

25. Hoagland DR, Arnon DI. The agua-culture method for growing plantas without soil. Californnia: Californnia Agricultural Experimental Station; 1950. 32 p.

26. Pereira FJ, de Castro EM, Pires MF, de Oliveira C, Pasqual M. Anatomical and physiological modifications in water hyacinth under cadmium contamination. J Appl Bot Food Qual. 2017;90:10-17, doi: 10.5073/JABFQ.2017.090.003

27. Ribani M, Bottoli CBG, Collins CH, Jardim ICSF, Melo LFC. Validação em métodos cromatográficos e eletroforéticos. Quim Nova. 2004 Sep/Oct;27(5):771-780, doi: 10.1590/S0100-40422004000500017

28. Writer JH, Ryan JN, Keefe SH, Barber LB. Fate of 4-nonylphenol and 17ß-estradiol in the Redwood River of Minnesota. Environ Sci Technol. 2011 Dec;46(2):860-868, doi: 10.1021/es2031664

29. Petrie B, Barden R, Kasprzyk-Hordern B. A review on emerging contaminants in wastewaters and the environment: current knowledge, understudied areas and recommendations for future monitoring. Water Res. 2015 Apr;72:3-27, doi: 10.1016/j.watres.2014.08.053

30. Agostini E, Talano MA, González PS, Oller ALW, Medina MI. Application of hairy roots for phytoremediation: what makes them an interesting tool for this purpose? Appl Microbiol Biotechnol. 2013 Feb;97(3):1017-1030, doi: 10.1007/s00253-012-4658-z

31. Hoang TTT, Tu LTC, Le NP, Dao QP. A preliminary study on the phytoremediation of antibiotic contaminated sediment. Int J Phytoremediation 2013 Mar;15(1):65-76, doi: $10.1080 / 15226514.2012 .670316$ 